\title{
Motor facilitation during action observation: the role of M1 and PMv in grasp predictions.
}

\author{
Toon T de Beukelaar ${ }^{1}$, Kaat Alaerts ${ }^{1,2}$, Stephan P Swinnen ${ }^{1}$, Nicole Wenderoth ${ }^{1,3}$ \\ ${ }^{1}$ Movement Control and Neuroplasticity Research Group, Dept. Kinesiology, KU Leuven, Belgium \\ ${ }^{2}$ Research Group for Neuromotor Rehabilitation, Dept. Rehabilitation Sciences, KU Leuven, Belgium \\ ${ }^{3}$ Neural Control of Movement Group, Department of Health Sciences and Technology, ETH Zurich, Switzerland
}

\section{ABSTRACT}

Recent theories propose that movement observation is not a "passive mirror" of ongoing actions but might induce anticipatory activity emerges when predictable movements are observed, e.g. because the action goal is known. Here we investigate this mechanism in a series of 3 experiments, by applying transcranial magnetic stimulation (TMS) to primary motor cortex (M1) while subjects observed either whole hand or precision grasping performed by an actor. We show that corticomotor excitability changes in a grip-specific manner but only once the grip can be decoded based on the observed kinematic cues (Exp. 1). By contrast, presenting informative contextual precues evokes anticipatory modulations in M1 already during the reach phase, i.e. well before the grip type could be observed, a finding in line with a predictive coding account (Exp. 2). Finally, we used paired-pulse TMS to show that ventral premotor cortex (PMv) facilitates grip-specific representations in M1 but only while grip formation is observed. These findings suggest that PMv and M1 interact temporarily and mainly when motor aspects of hand-object interactions are extracted from visual information. By contrast, no sustained input from $\mathrm{PMv}$ to $\mathrm{M} 1$ seems to be required to maintain action representations that are anticipated based on contextual information or once the grip is formed (Exp. 3).

Keywords: Human, movement observation, primary motor cortex, transcranial magnetic stimulation, ventral premotor cortex 
Abbreviations: WHG: Whole Hand Grip; PG: Precision Grip, TMS: transcranial magnetic stimulation, M1: primary motor cortex, $P M v$ : ventral premotor cortex, SMA: supplementary motor area, IPC: inferior parietal cortex, AIP: anterior intraparietal area 


\section{INTRODUCTION}

Movement observation activates the observer's motor cortex, a phenomenon mediated by so called 'mirror neurons'. Mirror neurons were first discovered in the ventral premotor cortex (PMv) (area F5) of the macaque monkey and were shown to fire while the monkey executes a specific action, but also when the monkey merely observes the same action performed by others (Di Pellegrino et al., 1992; Fadiga et al., 1995, 2005; Rizzolatti et al., 1996; Rizzolatti \& Craighero, 2004). Using a variety of neuroimaging and neurophysiological techniques, a similar 'mirror mechanism' has been demonstrated in the human brain, which includes, in addition to PMv, also the dorsal premotor cortex (PMd), supplementary motor area (SMA), primary motor cortex (M1) and the inferior parietal cortex (IPC) (Fadiga et al., 1995; Hari et al., 1998; Hamzei et al., 2003; Calmels et al., 2006; Kilner et al., 2009; Molenberghs et al., 2012; Kilner \& Lemon, 2013). In the past, studies using single-pulse transcranial magnetic stimulation (TMS) to measure changes in corticomotor excitability in M1 during movement observation have consistently demonstrated that observation-induced changes in excitability are highly specific to the actual muscles involved in the observed movement and occur time-locked to the observed kinematics while the movement unfolds (Fadiga et al., 1995; Gangitano et al., 2001; Alaerts et al., 2009a, 2009b, 2012; Koch et al., 2010). While most neurophysiological studies focused on M1, several lines of research have shown that PMv is a core area, upstream from M1, for mediating visual-to-motor transformations during both action execution and observation (e.g. Davare et al., 2006, 2008, 2010; Lago et al., 2010). Both in primates and humans, PMv-M1 interactions are modulated during movement execution and action preparation, which is indicative of a strong influence from PMv over M1 during these processes (Jeannerod et al., 1995; Murata et al., 1997; Binkofski et al., 1999; Grezes et al., 2003; Davare et al., 2006, 2008, 2009, 2010; Raos et al., 2006; Umilta et al., 2007).

Human PMv-M1 interaction can be probed using paired-pulse TMS such that a sub-threshold conditioning stimulus is applied over PMv that is shortly followed by a supra-threshold test stimulus 
applied over M1. This paired-pulse paradigm measures effective connectivity, i.e. the influence that one neural system exerts over another (Friston, 2011). It probes changes in M1 excitability that originate from the modified input from PMv and therefore provide insights into the activation of these cortico-cortical pathways (Koch \& Rothwell 2009; Lago et al., 2010). Using this paired-pulse TMS technique, Davare et al. $(2008,2010)$ tested PMv-M1 connectivity and showed that PMv specifically facilitates M1 in a muscle-specific fashion during the preparation and execution of grasping movements but not during rest.

Similar PMv-M1 interactions have been demonstrated during action observation. Particularly, disrupting PMv activity by repetitive TMS (rTMS) was shown to abolish the typical observationinduced increase in M1 corticomotor excitability (Avenanti et al., 2007). Also paired-pulse TMS paradigms have demonstrated modulations in PMv-M1 connectivity during action observation. For example, a study by Lago et al. (2010) showed that the M1 excitability increases in parallel with PMvM1 connectivity when observing a naturalistic grasping movement (e.g. human hand grasping a ball). Observing a noxious grasping movement (e.g. grasping a hot soldering iron) on the other hand, triggered a decrease in the strength of the PMv-M1 connectivity. Similarly, Koch et al. (2010) showed that PMv-M1 connectivity is specifically modulated when 'successful' goal directed reach-to-grasp actions are observed (i.e., actions in which the observed grasping posture is congruent with the goal of the action). No such change in PMv-M1 connectivity was shown for observing 'unsuccessful' actions, (i.e., actions in which the observed grasping posture is incongruent with the goal of the action).

Movement observation is not simply a passive perceptual process. Rather it fits within a predictive coding framework (Kilner et al., 2007), suggesting that observed actions are encoded at different levels: the intention level, the goal level, the kinematic level and the muscle level. Therefore, in order to fully 'understand' or make a prediction about an observed action, information at these different levels needs to be compared and combined in order to minimize potential prediction errors. For example, if at the highest level, a prediction about the intention of a perceived action is formed, this 
information will be used to create a prediction at the kinematic level which will be compared to the actual kinematics once the observed action starts to unfold. This comparison will result in a prediction error which in turn will be used to revise and improve the proposed intention. By repeating this process at different levels of the cortical hierarchy, predictions about observed actions can be optimized.

In summary, it has been shown that goal-encoding of the to-be-performed action during movement execution occurs prior to actual movement initiation, as reflected in M1 excitability changes during movement preparation. Moreover, it has been shown that PMv facilitates M1 in a muscle-specific fashion while preparing and executing grasping movements (Davare et al., 2008, 2010). Here we investigated whether similar anticipatory motor activity can be demonstrated during mere movement observation, and whether this phenomenon is modulated by input from PMv. Exploring the dynamic interplay between PMv and M1 will further our understanding of how contextual cues versus observed kinematics influence muscle-specific encoding in the motor system.

To explore these processes, we performed a series of three experiments measuring corticomotor excitability of the index finger (first dorsal interosseus, FDI) and the little finger (abductor digiti minimi, ADM) during the observation of two types of grasping actions; a precision and a whole hand grip.

In the first experiment, we show that excitability changes in $M 1$ are highly specific to the type of grasping action observed, allowing to decode whether a whole hand or precision grip is seen.

In the second experiment, we show that informative 'precues' that are available prior to movement initiation and that provide information on the type of the upcoming grasp are sufficient to modulate M1 excitability in a way consistent with predictive coding of the upcoming movement, i.e. a gripspecific facilitation pattern is already present in M1 before actual grip-specific kinematic information is observed. 
Finally, in the third experiment, paired-pulse TMS is used to test PMv-M1 interactions during anticipatory and online movement observation. We show that grip-specific muscle representations are facilitated via PMv-M1 pathways but only during the online observation of the initial phases of grip formation and not during the anticipatory prediction phase (i.e. prior to movement initiation) or during later phases of the observed movement (i.e. when the observed action is fully revealed). 


\section{MATERIALS AND METHODS}

\subsection{Participants}

13 subjects participated in Experiment 1 ( 3 males and 10 females, mean \pm SD age $26.1 \pm 4.9$ years), 11 in Experiment 2 ( 7 males and 4 females, age $21.5 \pm 1.1$ years) and 16 in Experiment 3 (6 males and 10 females, age $24.0 \pm 4.5$ ). All participants were right-handed, as assessed with the Edinburgh Handedness Questionnaire (Oldfield, 1971), naive about the purpose of the experiment and none of them had overt neurological disorders or impaired vision. Subjects were not allowed to participate in more than one experiment. The experimental procedure was approved by the local Ethics Committee for Biomedical Research at the Katholieke Universiteit Leuven in accordance to The Code of Ethics of the World Medical Association (Helsinki, 1964). All subjects were screened for adverse reactions to TMS and signed a written informed consent prior to participation when they complied with the inclusion criteria.

\subsection{General Setup}

In all three experiments, corticomotor excitability was measured using transcranial magnetic stimulation (TMS) during movement observation (Fig. 1).

Participants sat on a chair in front of a computer screen on which video-clips were displayed. Their right hand was placed on an armchair to ensure arm relaxation during the entire experimental session. In all three experiments, subjects observed video-clips showing right hand grasping actions performed by a female actor. Detailed information on the presented video-clips is provided separately for each experiment.

\subsection{Electromyographic recordings and TMS}

Focal TMS was performed by means of a $70 \mathrm{~mm}$ figure of eight coil connected to a Magstim 200 stimulator (Magstim, Whitland, Dyfed UK). The coil was positioned over the primary motor cortex (M1) of the left hemisphere, tangentially to the scalp with the handle pointing backwards and laterally at $45^{\circ}$ away from the mid-sagittal line, such that the induced current flow was in a posterior 
anterior direction, i.e. approximately perpendicular to the central sulcus (Pascual-Leone et al., 2002). The optimal scalp position ("hotspot") was defined as the position from which motor evoked potentials (MEP) with maximal amplitude were recorded in the right first dorsal interosseous (FDI). The rest motor threshold (rMT) was defined as the lowest stimulus intensity evoking MEPs in the right FDI with an amplitude of at least $50 \mu \mathrm{V}$ in 5 out of 10 consecutive stimuli (Rossini et al., 1994). Subjects' rMT, expressed as a percentage of the maximum stimulator output, varied in the 3 experiments with a mean of 45.2\% in Experiment 1 (33\% - 61\%), 39.7\% in Experiment 2 (34\% - 52\%) and $44.3 \%$ in Experiment $3(29 \%-60 \%)$. Though parameter setting procedures were prioritized for FDI, MEPs were simultaneously obtained for the abductor digiti minimi (ADM). ADM stimulation parameters were assumed to be satisfactorily similar, due to overlapping representations of hand muscles (Marconi et al., 2007) and the generally low threshold of the hand muscles. Signal Software (4.00 Version, Cambridge Electronic Desing, UK) was used for TMS triggering and electromyogram (EMG) recordings.

Disposable Ag-AgCl surface electrodes (Blue sensor SP Surface) were used to record an EMG from the FDI and ADM muscles. For each muscle the first electrode was placed on the belly, the second on the tendon of the according muscle and a third on a bony part (= reference electrode). The signals were sampled at $5000 \mathrm{~Hz}$ (CED Power 1401, Cambridge Electronic Design, UK) amplified, band-pass filtered (5-1000 Hz), and stored on a PC for offline analysis. Pre-stimulus EMG recordings were used to assess the presence of unwanted background EMG activity in the $110-10$ ms time interval preceding the magnetic pulse.

In experiment 1 and 2, single-pulse TMS was applied using a stimulation intensity of $130 \% \mathrm{rMT}$, as described previously (Alaerts et al., 2011).

In experiment 3, a paired-pulse TMS paradigm was applied to test whether muscle-specific modulations observed in M1 during the observation of grasping are facilitated/inhibited by the ventral premotor cortex (PMv). In 50\% of the trials, paired-pulse TMS was applied, by providing a 
sub-threshold conditioning stimulus (CS) over PMv (at $80 \%$ of the subjects' rMT), followed by a test stimulus (TS) applied over left M1 at $120 \%$ of the subjects' rMT (as described in Davare et al., 2008). In the remaining $50 \%$ of the trials, only the TS was applied over M1 which corresponds to single-pulse TMS applications. The inter stimulus interval between CS and TS was set at $7 \mathrm{~ms}$ (Davare et al., 2008). The CS over PMv was applied with a smaller TMS figure of eight coil $(50 \mathrm{~mm})$.

To determine the location of the PMv on the individual subjects' skull, a high resolution T1-weighted structural image was acquired for all subjects using a magnetization prepared rapid gradient echo sequence (MPRAGE; resolution time $(\mathrm{RT})=2300 \mathrm{~ms}$, echo time $(\mathrm{ET})=2.98 \mathrm{~ms}, 1 \times 1 \times 1.1 \mathrm{~mm}$ voxels, field of view (FOV): $240 \times 256,160$ sagittal slices). PMv was localized based on mean Montreal Neurological Institute (MNI) coordinates $-58,13,21(x, y, z)$ (averaging coordinates from Avenanti et al., 2007; Urgesi et al., 2007; and Davare et al., 2008, 2010) which were transformed into individual subjects space. The structural image was loaded into a 3D TMS neuronavigation system (Visor 1.0) and coregistered to the subjects' skull such that the coil position was superimposed on the structural image allowing online navigation to PMv.

\subsection{Stimuli}

Experiment 1. Stimuli consisted of two video-clips showing the grasping and lifting of a round jar (9 $\mathrm{cm}$ diameter, $14.5 \mathrm{~cm}$ height, $1.5 \mathrm{~kg}$ weight) either with a whole hand grip (WHG) or precision grip (PG) (Fig. 2A). During the whole hand grip all fingers were used to grasp the lid of the jar, whereas for the precision grip, only the thumb and index finger grasped a small knob mounted on the top of the jar. Before the start of either video-clip the screen was black. At the start of the clip the black screen disappeared and a metal jar was visible in the middle of the screen. Then the right hand of an actor came into view in the upper right corner as a clenched fist. Importantly, up until the opening of the fist the movements were identical in both video-clips. The grip type only became apparent after the initial reaching phase. The jar was grasped and lifted approximately $15 \mathrm{~cm}$ above the surface. In total 20 movies were shown, each consisting of a series of 10 grasping movements. In each movie series, 
we randomized the occurrence of whole hand grip and precision grip to avoid anticipation on the type of grasp by the observing participants. In half of the video-clips, a precision grip ( $n=100)$ was shown, whereas in the other half, a whole hand grip was shown $(n=100)$.

During the observation of a grasping movement (whole hand or precision grip) a single TMS pulse was administered randomly at one of five phases in the movement sequence: (i) during the reach phase showing the actor's hand as a clenched fist ( $2 \mathrm{~s}$ after initiation of the video); (ii) during the open phase when the hand opened up to initiate the grasping (2.6 s after initiation); (iii) during the grasp phase when the hand forms according to the grip to be performed (3.48 $\mathrm{s}$ after initiation); (iv) during the contact phase when the fingers make contact with the jar (3.76 $\mathrm{s}$ after initiation); and finally ( $v$ ) during the lift phase (4.92 s after initiation). Twenty MEPs were recorded for each of the ten conditions ( 2 grip types (WHG, PG) x 5 stimulation points (reach, open, grasp, contact, lift)), resulting in 200 MEPs in total for each subject and each muscle (FDI, ADM). To ensure that participants paid attention to the movies a one-back task was performed such that participants had to say after each movement, whether the observed type of grip was the same as the one shown in the previous clip or not.

The complete analysis of the dataset is presented in the supplementary material (Suppl. Fig. 1). Nevertheless, to keep the analysis consistent with experiments 2 and 3, we limit our further analysis to the data collected during the reach, grasp and lift phase. Therefore we consider 6 conditions ( 2 grip types (WHG, PG) x 3 stimulation points (reach, grasp, lift)), and in total 120 MEPs for each subject and each muscle (FDI, ADM) in our further analysis of Experiment 1.

Experiment 2. The effect of a 'precue', presented before the actual video-clip which provided information regarding the type of the upcoming grasp action, was investigated. Specifically, at the start of half of the video-clips, either a blue or white square was presented on the screen, indicating that the upcoming grasping action would involve a whole hand or precision grip, respectively (Fig. 2B). In the other half of the video-clips, no precue was applied before movement observation was 
initiated. During the observation of each video-clip, a single TMS pulse was administered (i) during the reaching phase showing the actor's hand as a clenched fist ( $2 \mathrm{~s}$ after initiation of the video) or (ii) during the lift phase (4.92 s after initiation).

To ascertain the participants' attention to the precues and upcoming grips, they had to indicate after each video-clip whether the presented precue corresponded with the observed grip type. Participants were informed that the percentage of incorrect precues was rare ( $8.3 \%$ of all trials), but that it was important to identify them correctly.

In total 20 movies were shown, each consisting of a series of 8 grasping movements. For each of the eight conditions (2 grip types (WHG, PG) $\times 2$ stimulation points (reach, lift) $\times 2$ types of precue (no precue, precue)), 20 MEPs were recorded, resulting in a total of 160 MEPs for each subject and this for each muscle (FDI, ADM).

Experiment 3. Stimuli were identical to those of Experiment 2, i.e. all video-clips were preceded by a precue, providing information on the upcoming grip, (i.e., blue square for WHG; white square for PG) (Fig. 2C) and participants were instructed to indicate after each video-clip whether the presented precue corresponded to the observed grip type. Incorrect precues were rare, but present $(10 \%)$ to ensure that subjects paid attention.

While observing the video-clips, a TMS test-stimulus (TS) was administered (i) during the reaching phase ( $2 \mathrm{~s}$ after initiation of the video); (ii) during the grasp phase (3.48 $\mathrm{s}$ after initiation); or (iii) during the lift phase (4.92 s after initiation). In half of the trials, the test-stimulus was preceded by a conditioning stimulus (CS), administered in a randomized order.

In total 20 movies were shown, each consisting of a series of 12 grasping movements. For each of the 12 conditions ( 2 grip types (WHG, PG) x 3 stimulation points (reach, grasp, lift) $\times 2$ stimulation types (single-pulse, paired-pulse)), 20 MEPs were collected, resulting in a total of 240 collected MEPS for each subject and each muscle (FDI, ADM). 
At the start of the experimental session, the effects of single- and paired-pulse TMS were measured during 'rest' and without observing any stimuli. To do so, subjects sat relaxed with their eyes closed for 3 min, during which 24 test-stimuli were administered over M1. Half of these test-stimuli were preceded by a conditioning stimulus over PMv to obtain a control measure of M1-PMv interactions during rest.

\subsection{Data Analysis and Statistics}

Peak-to-peak amplitudes of the MEPs were determined from the EMG data. The MEP amplitude is known to be modulated by EMG background activation (Devanne et al., 1997; Hess et al., 1987). Therefore pre-trigger EMG recordings were used to assess the presence of unwanted background EMG activity in the 10-110 ms preceding the magnetic pulse and were calculated by computing the root mean square scores (RMS) across this interval. For each subject we calculated the mean and standard deviations of the background EMG over all trials. MEPs were excluded when preceded by background EMG higher than $0.01 \mathrm{mV}$. Trials were also removed from further analysis if pre-trigger EMG exceeded the mean +2.5 standard deviations. Furthermore we considered MEP peak-to-peak amplitudes which exceeded Q3 + 1.5 x (Q3 - Q1), with Q1 being the first quartile and Q3 the third quartile computed over the whole set of trials for each subject, as outliers and were therefore removed from further analysis. Following these criteria, a total of $3.3 \%$ of all trials were excluded from further analysis.

To determine whether M1 excitability was modulated in a grip-specific manner, a ratio was calculated by dividing the MEP mean amplitude collected while observing a whole hand grip by the MEP mean amplitude for observing a precision grip (ratio $=\mathrm{MEP}_{\mathrm{WHG}} / \mathrm{MEP}_{\mathrm{PG}}$ ). This was done separately for each muscle (FDI, ADM). Accordingly, a ratio higher than 1 indicates a stronger facilitation during whole hand grip observation while a value lower than 1 , indicates a stronger facilitation during precision grip observation. A ratio equal to 1 indicates no muscle-specific 
facilitation/inhibition. Ratios were calculated for each subject and condition and subjected to statistical analyses.

Experiment 1. To test the extent of muscle-specific facilitation in M1 during whole hand versus precision grip observation, the ratio scores ( $\mathrm{MEP}_{\mathrm{WHG}} / \mathrm{MEP}_{\mathrm{PG}}$ ) were subjected to a repeated measures ANOVA with the within-subject factors muscle (FDI, ADM) and phase (Reach, Grasp, Lift). In addition, we tested whether MEP ratios differed significantly from 1. Since we had a strong a-priory hypothesis, we used one-sided t-tests to determine whether MEP ratios are significantly $>1$ for the $A D M$ and $<1$ for the FDI as predicted for grip-specific modulation.

Experiment 2. Based on the results of Experiment 1, we specifically tested the hypothesis whether a precue indicating the upcoming grasp is sufficient to modulate M1 excitability during the reaching phase. To do so, we specifically tested whether a muscle (FDI, ADM) by cue (cue, no-cue) interaction was evident during the reach phase. If a contextual cue indicating the upcoming action can modulate M1 excitability already during the reaching phase we expect to find a muscle effect only in the 'cue' condition, and not in the 'no-cue' condition (i.e., a significant muscle by cue interaction).

As a control, similar dynamics were explored during the lift phase, albeit here, muscle-specific M1 excitability modulations are expected irrespective of whether a precue was provided or not (i.e., as reflected by a significant main effect of muscle, not a muscle by cue interaction).

Experiment 3. To explore the facilitatory effect of a conditioning stimulus over PMv on musclespecific facilitation in M1, we calculated ratio scores per muscle by dividing the paired-pulse (PP) TMS data by the single-pulse (SP) TMS data (MEPPP/MEPSP) for both the whole hand grip and precision grip in each movement phase. T-tests were performed to directly explore whether pairedpulse/single-pulse ratios are significantly higher than 1 (facilitatory effect).

Statistics were calculated with Statistica 8 (StatSoft, USA) and results with a p-value lower than 0.05 were considered significant. 


\section{RESULTS}

\subsection{Experiment 1}

Here we show that muscle-specific facilitation is seen in M1 when observing a whole hand versus a precision grip (Fig. 3A; Suppl. Table 1). More specifically this effect was seen during the grasp and lift phase, but did not emerge during the reach phase when no grasp-specific information is available to the observer. The repeated measures ANOVA revealed a significant muscle by phase interaction $\left(F(2,24)=4.59, p<0.05, \eta^{2}=0.28\right)$. Furthermore, a main effect for muscle was found $(F(1,12)=6.74$, $\left.p<0.05, \eta^{2}=0.36\right)$, but not for phase $\left(p=0.32, \eta^{2}=0.09\right)$.

In the ADM muscle we found that MEP ratio scores were significantly higher than 1 in the grasp and lift phase (grasp phase: $t=1.91$, lift phase: $t=2.27 ; p<0.05$ ) indicating that during these phases, ADM was facilitated more strongly by whole hand grip observation than by precision grip observation. For the FDI muscle, a significantly lower value than 1 was found for the lift phase $(t=-$ $1.91, p<0.05)$ indicating more M1 facilitation of the FDI for precision grip observation than for whole hand grip observation. The more detailed evolution of grip-specific facilitation patterns can be found in supplementary figure 1.

In summary, our data of experiment 1 show that FDI and ADM are differentially modulated when observing a precision versus whole hand grip, and that grasp-specific facilitation only emerges when the movement kinematics unfold during the grasp and lift phase. No such muscle-specific modulation was observed during the reach phase, during which no grasp-specific information on the upcoming grasping movement was presented.

\subsection{Experiment 2}

Here we show that a precue, providing information about the upcoming 'to-be-observed' grasping movement, evokes anticipatory muscle-specific facilitation in M1 (Fig. 3B, left panel; Suppl. Table 2). More specifically, during the reach phase, MEP ratio scores were close to 1 for both FDI and ADM when no informative cue was provided, thus replicating results from experiment 1 . By contrast, in 
trials where an informative cue was present, M1 excitability was modulated in a grip-specific way, which was confirmed by a significant muscle by cue interaction $\left(F(1,10)=3.39, p<0.05, \eta^{2}=0.25\right)$. This effect was particularly pronounced for the ADM with ratios clearly exceeding 1 ( $t=1.92, p<$ 0.05), thus indicating that ADM was more strongly facilitated when the whole hand grip than when the precision grip was anticipated. A similar tendency was observed for the FDI such that MEP ratio scores were on average $<1$ (indicating stronger facilitation for observing a precision versus power grip), however, this effect did not reach significance $(p=0.11)$.

For the lift phase, muscle-specific modulations were present irrespective of whether or not a precue was presented, thus replicating results from Experiment 1 . This was confirmed by a control analysis using a separate repeated measures ANOVA model for the lift phase that revealed a significant muscle main effect $\left(\mathrm{F}(1,10)=8.28, \mathrm{p}<0.05, \eta^{2}=0.45\right)$ but no muscle by cue interaction $(\mathrm{F}(1,10)=$ $\left.0.0023, p=0.48, \eta^{2}<0.01\right)$

Overall, these results indicate that when an informative cue is presented prior to the observation of a specific grasping action, anticipatory muscle-specific facilitation is observed even before actual movement information is presented. We also found indications that within our experimental set-up this effect is more pronounced for the ADM compared to the FDI.

\subsection{Experiment 3}

We investigated the role of PMv on muscle-specific facilitation seen in M1 during the observation of a specific grasping task (Suppl. Table 3).

We first measured the overall PMv-M1 connectivity during rest and found that a general inhibition is exerted from PMv towards M1, resulting in a decrease in corticomotor excitability measured when comparing paired-pulse TMS (PMv-M1) to single pulse (M1) (MEP $\mathrm{PP}_{\mathrm{PP}} / \mathrm{MEP}_{\mathrm{SP}}=0.92 \pm 0.23$ averaged across muscles; $\mathrm{t}=-1.71 ; \mathrm{p}<0.05)$.

Single-pulse TMS data (Fig. 4A) replicated the findings of experiment 1 and 2, i.e. grip-specific facilitation of the ADM and FDI was observed for the reach and lift phase (muscle main effect; reach 
phase: $F(1,15)=17.32, p<0.01, \eta^{2}=0.54$; lift phase: $\left.F(1,15)=18.21, p<0.01, \eta^{2}=0.55\right)$. Surprisingly, we did not find this pattern of results during the grasp phase (no muscle main effect; $F(1,15)=0.76, p$ $\left.=0.20, \eta^{2}=0.05\right)$.

Next we specifically explored whether PMv-M1 connectivity changes during the different phases of movement observation (Fig. 4B). We found PMv-M1 modulation for the ADM during the grasp phase, more specifically $M E P_{p p} / M E P_{s p}$ was significantly larger when observing the whole hand grip than when observing the precision grip $(t=3.74, p<0.01)$ and this grip-specific modulation was found in 14 out of 16 subjects. For this condition, $A D M M E P_{p p} / M E P_{s p}$ differed significantly from $1(t=2.72, p<$ 0.05) when observing a whole hand grip suggesting that PMv specifically facilitated ADM representations in M1 while observing how a whole hand grip was formed. By contrast no PMv-M1 facilitation was found when the formation of a precision grip was observed. This result was specific to the ADM and to the grasp phase since no other significant effect was found. 


\section{DISCUSSION}

We conducted a series of three experiments to measure muscle-specific modulations in M1 during the observation of precision versus whole hand grasping actions. Corticomotor excitability was measured (i) prior to grasp initiation (reach phase); (ii) during the grasp phase; or (iii) during the lift phase. Movement observation modulated corticomotor excitability during the grasp and lift phase such that facilitation of FDI versus ADM was consistent with grip-specific muscle facilitation patterns for whole hand versus precision grips (Davare et al., 2008). However, overall, grip-specific modulation was stronger in the ADM than in the FDI. In the second experiment we showed that contextual cues evoke grip-specific changes in corticomotor excitability even before the grip type could be observed in the video, suggesting that the M1 activity observed here is in line with a predictive coding framework. Finally, we tested PMv-M1 connectivity and found that particularly ADM is facilitated in a grip-specific manner but only while the grip formation is observed.

Across all three experiments, we replicated prior findings of muscle-specific modulation of M1 during mere movement observation of the lift phase (Alaerts et al., 2009a, 2009b, 2010, 2011; Fadiga et al., 1995, Koch et al., 2010). Most importantly, the muscle specific facilitation pattern measured during movement observation was highly consistent with grip-specific facilitation reported for movement preparation and execution (Davare et al., 2008). Moreover, it changed dynamically while the grasping action unfolded such that characteristic facilitation patterns were linked to the specific phase of the observed movement. When the subject had no prior information regarding the upcoming grip type (Exp. 1), significant grip-specific modulation was found when the grip formation was observed but not before (Suppl. Fig. 1). During this grasping phase, grip-specific modulation of corticomotor excitability was substantial for ADM but only minor for FDI. This might be due to the fact that the ADM solely contributes to a whole hand grip and not to a precision grip formation. The FDI on the other hand contributes to the formation of both the whole hand and precision grip, which might lead to less distinct excitability patterns. 
Considering the lift phase of whole hand and precision grip actions, a more prominent musclespecific facilitation occurs for ADM and FDI, which is in line with previous research (Janssen et al., 2013, Mc Cabe et al., 2014, Senot et al., 2011). Particularly, prior research from our lab showed that corticomotor excitability is higher when observing the lifting of a heavy object (requiring to exert more force), compared to observing the lifting of a light object (requiring to exert less force) (Alaerts et al., 2011). Our findings are in line with these results, since during the lifting of the object more force is produced by the FDI and ADM, which will amplify the muscle-specific facilitation that has been initiated during the grasping action. In both experiments subjects performed a behavioral control task to ensure that they were alert throughout testing. However, this task could be solved as soon as the grip kinematics had been observed, which leads to the possibility that the subject's attention might have been lower during the subsequent phases of the observed movement. Even though we cannot exclude that attention varied during a trial, previous research has shown that the observation-induced modulation of MEP responses is only minimally affected by attention based demands (as long as no visual distracters are used) (Saucedo Marquez et al., 2011).

In our second and third experiment we presented informative 'precues' regarding the upcoming grip type (precision versus whole hand) prior to observing the actual action to investigate anticipatory muscle-specific modulation in M1. Interestingly, we found that the presentation of a precue induced muscle-specific facilitation already during the early reach phase. These findings indicate that a predictive cue can activate motor representations in M1 according to the anticipated to-be-observed grasp, even before the actual kinematics are visible.

These findings are in line with Alaerts et al. (2011) who demonstrated that blocked presentations of movement videos can induce 'anticipatory' excitability changes. This prior study showed that observing a heavier object generally results in a higher corticomotor excitability for the contributing muscles but, interestingly, that this force encoding was already present during the early reach phase at which no indicative cues on the weight of the object were available to the subjects. These data therefore suggested that anticipatory M1 excitability was scaled according to the weight of the 
object experienced in the previous lift when no external input related to the object's weight was available.

Our results can also be interpreted within a framework of predictive coding during movement observation. Kilner et al. (2004) showed that motor areas are activated when observing a specific movement, but more intriguingly that such motor activity can also occur before the action is visible. This study therefore provided evidence that the motor system is activated in correspondence to the goal of an upcoming movement, which makes it possible to not only react but also to anticipate observed actions made by others. Various studies have shown similar results, indicating that a motor plan can be primed and influences the state of the motor system (Alaerts et al., 2011; Craighero et al., 2008; Grafton et al., 1997; Mc Cabe et al., 2014; Tucker \& Ellis, 1998; Urgesi et al., 2006).

More specifically, Mc Cabe et al. (2014) investigated the time course of the corticomotor excitability during the observation of a grasping task. Similar to our findings during the reach phase, when the goal of the upcoming grasping action was known before onset, this knowledge influenced the state of the motor system in a muscle-specific fashion. On the other hand, when no information on the goal of the action was provided beforehand, no muscle-specific excitability was observed prior to movement onset. Furthermore and in line with our results of the lift phase, this study shows that whether or not information about the upcoming movement was provided, a kinematic modulation of the corticomotor excitability occurred when the action unfolded.

Finally, in our third experiment, we investigated the facilitatory/inhibitory role of PMv over M1 during the observation of the reaching-grasping-lifting action.

Replicating our findings in Experiment 1 and 2, we found in experiment 3 that muscle-specific M1 facilitation, as measured by single-pulse TMS, was evident during both the reach phase (i.e., when the informative precue was provided) and the lift phase (i.e., when the grasping movement was finalized). However during the grasp phase, grip-specific muscle facilitation was only minor. At first sight this seems to be inconsistent with our results in Experiment 1 but it is important to note that 
the video-clips in Experiment 3 were preceded by informative precues which might have caused the divergent excitability pattern during the grasping phase. This lack of a strong muscle-specific facilitation pattern in the grasp phase is consistent with previous findings of Loh et al. (2010) and Alaerts et al. (2011). Alaerts et al. (2011) used a paradigm where an actor lifted either heavy or light objects. Importantly, subjects knew that the same movement stimulus would be shown repeatedly such that the upcoming force requirements could be predicted. This study showed that musclespecific facilitation patterns are observed during the reach phase (consistent with a predictive coding account proposing that knowledge of an action goal activates motor representations and kinematics in a predictive manner) but that excitability of $\mathrm{M} 1$ is reduced when the grip formation is observed (their Fig. 2a). Therefore our data in combination with the work of Loh et al. (2010) indicate that visual cues can cause a rapid updating of motor representations in M1. Note that M1-stimulation intensities differed across experiments (130\% rMT in experiment 1 and 120\% rMT in experiment 3), however, it is unlikely that this relatively small difference would explain the absence of musclespecific MEP modulation during only one phase. Instead, we argue that once the actor's grasping kinematics are observable, this information is used to update the state of the observer's motor system in a manner consistent with a shift from prediction to processing available information. It is important to note that our experiment was designed such that the predicted and observed grasp kinematics were congruent in all analyzed trails. Therefore, we were not able to test the extent to which prediction errors (i.e. a discrepancy between predicted and observed movements) would influence the updating mechanism via PMv-M1 interactions described here. However, other studies used discrepancy paradigms in which subjects anticipate a specific type of grasping action based on the presentation of a contextual or symbolic cue, but observe a different type of grasping action. For example Cavallo et al. (2013) showed that an anticipatory motor plan is prepared in M1 based on the presentation of the goal of the action within a specific situational context. Once kinematic information was provided during the observation of the unfolding action, this novel information enabled an updating of the initial motor plan and correspondingly triggered a modulation of 
excitability changes measured in M1. A subsequent study by Janssen et al. (2013) provided further indications that modulation of corticomotor excitability during observation can be segmented, such that it progressed initially in accordance with the action anticipated and, if discrepancies became evident from the visual input, it subsequently coincided with that of the action seen.

Our paradigm was set up to test the influence of $\mathrm{PMv}$ over $\mathrm{M} 1$ during the unfolding phases of the observed action. Applying a conditioning stimulus over PMv prior to stimulating M1 revealed that PMv-M1 connectivity changed dynamically, depending on which movement phase was observed. Importantly, we found strong facilitatory connectivity between PMv and M1 specifically when subjects observed how the whole hand grip was formed.

Based on this pattern in the results, we propose that prior knowledge of the to-be-observed grip - as provided by a precue during the reach phase - can trigger anticipatory activation in the observers motor system predicting the upcoming movement (as in Experiment 2) but this does not require the sustained, facilitatory drive from PMv. Instead, PMv-M1 connectivity was specifically modulated at the beginning of the grasp phase, i.e. during the supposed switch from grip prediction to grip observation. This finding may therefore indicate a prominent role of PMv in facilitating muscle specific representations of M1 according to readily available - online emerging visual information, whereas during the formation of anticipatory representations (as measured during the reach phase) PMv seems less involved. The prominent role for PMv in kinematic encoding is in accordance with previous studies showing its crucial contribution in the activation pattern of the appropriate intrinsic hand muscles when grasping a specific object (Davare et al., 2006, 2009). Moreover single-cell recordings in behaving monkeys have revealed that PMv neurons with motor properties encode gripspecific information predominantly during movement execution and that firing rates of these PMV neurons are highest while the object is grasped (Fluet et al., 2010).

Along this line, it can be speculated that different brain regions will contribute to the anticipatory versus online encoding of observed movements by projecting either directly or indirectly to M1. 
Another area that might be involved in anticipating actions based on contextual cues or general goal representations is parietal cortex, particularly, the anterior intraparietal sulcus (AIP) (Hamilton et al., 2006; Davare et al., 2010). In previous research, the parietal area has been shown to form an integral part of the human mirror network in addition to PMv (for review see Rizzolatti and Craighero, 2004). During execution, Davare et al. (2010) demonstrated that the AIP provides information about object properties and drives grasp-related and muscle-specific PMv-M1 interactions. It would be interesting to explore whether similar dynamics are observed during movement observation. To do so, a pairedpulse paradigm can be envisaged that explores the modulatory role of AIP over M1 during movement observation. Moreover, investigating other brain regions' connectivity, e.g. regions known to contribute to grasp-related interactions during execution and/or being part of the human mirror neuron network, at different paired-pulse delays might further our understanding of M1 modulations during movement observation.

PMv-M1 interactions might be specifically geared towards extracting key elements of the 'online' observed action to identify which grip type is used by the actor. However, once an action representation is activated, $\mathrm{PMv}-\mathrm{M} 1$ interaction seems no longer necessary to maintain grip-specific facilitation in $\mathrm{M} 1$, hence the lack of continued PMv-M1 modulation in the ongoing lift phase. However, regions other than PMv might have exerted their modulatory effect on $\mathrm{M} 1$ in order to maintain grip-specific information. Note that we cannot exclude the possibility that presenting the precue already modulated PMv-M1 connectivity in a way similar to observing the actual grip formation since PMv-M1 connectivity was only tested after the precue was presented. However, previous work suggests that mapping arbitrary cues (as the color cues used in the present experiment) to movements relies predominantly on activity of PMd whereas PMv is believed to process direct cues (such as an object's geometrical properties) that are relevant for the upcoming grasp (Majdandžic et al., 2009; Chouinard \& Paus, 2010). 


\subsection{Conclusion:}

We show that muscle-specific activation in M1 is seen during movement observation. We also show that a precue presented before movement observation can activate motor representations of the predicted grasp, as revealed by identical muscle-specific facilitation as that observed during the observation of the actual movement. Finally, we show that action observation modulates PMv-M1 connectivity such that PMv facilitates muscle representations in $\mathrm{M} 1$ in accordance with the observed action and specifically during actual grip formation, but not during movement anticipation or final phases of the observed movement. We propose that PMv and M1 interact only temporarily when essential characteristics of the observed action are extracted in order to facilitate the appropriate muscular representation. Our data also suggest that increased excitability in M1, which is typically seen during action observation, does not require the continuous facilitatory drive from PMv. For future research, it would be interesting to explore how PMv influences muscle-specific encoding in M1 when the observed movement deviates from the anticipated action plan (similar to Janssen et al., 2013). 


\section{ACKNOWLEDGEMENTS}

This work was supported by grants from the Research Foundation - Flanders (grant numbers G.0401.12, 1521313N) and a grant from the Swiss National Science Foundation (project number 320030_149561). T.T.d.B. is a predoctoral fellow of the Research Foundation Flanders and K.A. is supported by an FWO postdoctoral research fellowship grant and by a Branco Weiss fellowship of the Society in Science - Eidgenössische Technische Hochschule Zurich. 


\section{REFERENCES}

Alaerts, K., Heremans, E., Swinnen, S. P., \& Wenderoth, N. (2009a). How are observed actions mapped to the observer's motor system? Influence of posture and perspective. Neuropsychologia, 47(2), 415-22. doi:10.1016/j.neuropsychologia.2008.09.012

Alaerts, K., Swinnen, S. P., \& Wenderoth, N. (2009b). Is the human primary motor cortex activated by muscular or direction-dependent features of observed movements? Cortex, 45(10), 1148-55. doi:10.1016/j.cortex.2008.10.005

Alaerts, K., Senot, P., Swinnen, S. P., Craighero, L., Wenderoth, N., \& Fadiga, L. (2010). Force requirements of observed object lifting are encoded by the observer's motor system: a TMS study. The European Journal of Neuroscience, 31(6), 1144-53. doi:10.1111/j.1460-9568.2010.07124.x

Alaerts, K., Swinnen, S. P., \& Wenderoth, N. (2011). Action perception in individuals with congenital blindness or deafness: how does the loss of a sensory modality from birth affect perception-induced motor facilitation? Journal of Cognitive Neuroscience, 23(5), 1080-7. doi:10.1162/jocn.2010.21517

Alaerts, K., de Beukelaar, T. T., Swinnen, S. P., \& Wenderoth, N. (2012). Observing how others lift light or heavy objects: time-dependent encoding of grip force in the primary motor cortex. Psychological Research, 76(4), 503-13. doi:10.1007/s00426-011-0380-1

Avenanti, A., Bolognini, N., Maravita, A., \& Aglioti, S. M. (2007). Somatic and motor components of action simulation. Current Biology, 17(24), 2129-35. doi:10.1016/j.cub.2007.11.045

Binkofski, F., Buccino, G., Posse, S., Seitz, R. J., Rizzolatti, G., \& Freund, H. J. (1999). A fronto-parietal circuit for object manipulation in man: evidence from an fMRI-study. The European Journal of Neuroscience, 11(9), 3276-86. 
Calmels, C., Holmes, P., Jarry, G., Lévèque, J.-M., Hars, M., \& Stam, C. J. (2006). Cortical activity prior to, and during, observation and execution of sequential finger movements. Brain Topography, 19(12), 77-88. doi:10.1007/s10548-006-0014-x

Cavallo, A., Bucchioni, G., Castiello, U., \& Becchio, C. (2013). Goal or movement? Action representation within the primary motor cortex. The European Journal of Neuroscience, 38(10), 3507-12. doi:10.1111/ejn.12343

Chouinard, P. A., \& Paus, T. (2010). What have We Learned from "Perturbing" the Human Cortical Motor System with Transcranial Magnetic Stimulation? Frontiers in Human Neuroscience, 4, 173. doi:10.3389/fnhum.2010.00173

Craighero, L., Bonetti, F., Massarenti, L., Canto, R., Fabbri Destro, M., \& Fadiga, L. (2008). Temporal prediction of touch instant during observation of human and robot grasping. Brain Research Bulletin, 75(6), 770-4. doi:10.1016/j.brainresbull.2008.01.014

Davare, M., Andres, M., Cosnard, G., Thonnard, J.-L., \& Olivier, E. (2006). Dissociating the role of ventral and dorsal premotor cortex in precision grasping. The Journal of Neuroscience, 26(8), 2260-8. doi:10.1523/JNEUROSCI.3386-05.2006

Davare, M., Lemon, R., \& Olivier, E. (2008). Selective modulation of interactions between ventral premotor cortex and primary motor cortex during precision grasping in humans. The Journal of Physiology, 586(Pt 11), 2735-42. doi:10.1113/jphysiol.2008.152603

Davare, M., Montague., K., Olivier, E., Rothwell, J. C., \& Lemon, R. N. (2009). Ventral premotor to primary motor cortical interactions during object-driven grasp in humans. Cortex, 45(9), 1-14. 
Davare, M., Rothwell, J. C., \& Lemon, R. N. (2010). Causal connectivity between the human anterior intraparietal area and premotor cortex during grasp. Current Biology, 20(2), 176-81. doi:10.1016/j.cub.2009.11.063

Devanne, H., Lavoie, B. A., \& Capaday, C. (1997). Input-output properties and gain changes in the human corticospinal pathway. Experimental Brain Research. 114(2),329-38.

Di Pellegrino, G., Fadiga, L., Fogassi, L., Gallese, V., \& Rizzolatti, G. (1992). Understanding motor events: a neurophysiological study. Experimental Brain Research. 91(1):176-80.

Fadiga, L., Fogassi, L., Pavesi, G., \& Rizzolatti, G. (1995). Motor facilitation during action observation: a magnetic stimulation study. Journal of Neurophysiology. 73(6):2608-11.

Fadiga, L., Craighero, L., \& Olivier, E. (2005). Human motor cortex excitability during the perception of others' action. Current Opinion in Neurobiology, 15(2), 213-8. doi:10.1016/j.conb.2005.03.013

Fluet, M.-C., Baumann, M. A., \& Scherberger, H. (2010). Context-specific grasp movement representation in macaque ventral premotor cortex. The Journal of Neuroscience, 30(45), 15175-84. doi:10.1523/JNEUROSCI.3343-10.2010

Friston, K. J. (2011). Functional and effective connectivity: a review. Brain Connectivity, 1(1), 13-36. doi: 10.1089/brain.2011.0008

Gangitano, M., Mottaghy, F. M., \& Pascual-Leone, A. (2001). Phase-specific modulation of cortical motor output during movement observation. Neuroreport, 12(7), 1489-92.

Grafton, S. T., Fadiga, L., Arbib, M. A., \& Rizzolatti, G. (1997). Premotor cortex activation during observation and naming of familiar tools. Neurolmage, 6(4), 231-6. doi:10.1006/nimg.1997.0293 
Grèzes, J., Armony, J. L., Rowe, J., \& Passingham, R. E. (2003). Activations related to "mirror" and "canonical" neurones in the human brain: an fMRI study. Neurolmage, 18(4), 928-37. doi:10.1016/S1053-8119(03)00042-9

Hamilton, A. F. C., \& Grafton, S. T. (2006). Goal representation in human anterior intraparietal sulcus. The Journal of Neuroscience, 26(4), 1133-7. doi:10.1523/JNEUROSCI.4551-05.2006

Hamzei, F., Rijntjes, M., Dettmers, C., Glauche, V., Weiller, C., \& Büchel, C. (2003). The human action recognition system and its relationship to Broca's area: an fMRI study. Neurolmage, 19(3), 637-44. doi:10.1016/S1053-8119(03)00087-9

Hari, R., Forss, N., Avikainen, S., Kirveskari, E., Salenius, S., \& Rizzolatti, G. (1998). Activation of human primary motor cortex during action observation: a neuromagnetic study. Proceedings of the National Academy of Sciences of the United States of America, 95(25), 15061-5.

Hess, C. W., Mills, K. R., \& Murray, N. M. F. (1987). Responses in small hand muscles from magnetic stimulation of the human brain. Journal of Physiology. 388,397-419.

Janssen, L., Steenbergen, B., \& Carson, R. G. (2013). Anticipatory Planning Reveals Segmentation of Cortical Motor Output During Action Observation. Cerebral Cortex. 25(1),192-201. doi:10.1093/cercor/bht220

Jeannerod, M., Arbib, M. A., Rizzolatti, G., \& Sakata, H. (1995). Grasping objects: the corticalmechanisms of visuomotor transformation. Trends in Neurosciences. 18(7):314-20.

Kilner, J. M., Vargas, C., Duval, S., Blakemore, S.-J., \& Sirigu, A. (2004). Motor activation prior to observation of a predicted movement. Nature Neuroscience, 7(12), 1299-301. doi:10.1038/nn1355

Kilner, J. M., Friston, K. J., \& Frith, C. D. (2007). Predictive coding: an account of the mirror neuron system. Cognitive Processing, 8(3), 159-66. doi:10.1007/s10339-007-0170-2 
Kilner, J. M., Neal, A., Weiskopf, N., Friston, K. J., \& Frith, C. D. (2009). Evidence of mirror neurons in human inferior frontal gyrus. The Journal of Neuroscience, 29(32), 10153-9. doi:10.1523/JNEUROSCI.2668-09.2009

Kilner, J. M., \& Lemon, R. N. (2013). What we know currently about mirror neurons. Current Biology, 23(23), R1057-62. doi:10.1016/j.cub.2013.10.051

Koch, G., \& Rothwell, J. C. (2009). TMS investigations into the task-dependent functional interplay between human posterior parietal and motor cortex. Behavioural Brain Research, 202(2), 147-52. doi:10.1016/j.bbr.2009.03.023

Koch, G., Versace, V., Bonnì, S., Lupo, F., Lo Gerfo, E., Oliveri, M., \& Caltagirone, C. (2010). Resonance of cortico-cortical connections of the motor system with the observation of goal directed grasping movements. Neuropsychologia, 48(12), 3513-20. doi:10.1016/j.neuropsychologia.2010.07.037

Lago, A., Koch, G., Cheeran, B., Márquez, G., Sánchez, J. A., Ezquerro, M., Giraldez, M., Fernández-delOlmo, M. (2010). Ventral premotor to primary motor cortical interactions during noxious and naturalistic action observation. Neuropsychologia, 48(6), 1802-6. doi:10.1016/j.neuropsychologia.2010.02.030

Loh, M. N., Kirsch, L., Rothwell, J. C., Lemon, R. N., \& Davare, M. (2010). Information about the weight of grasped objects from vision and internal models interacts within the primary motor cortex. The Journal of Neuroscience, 30(20), 6984-90. doi:10.1523/JNEUROSCI.6207-09.2010

Majdandžic, J., Bekkering, H., van Schie, H. T., \& Toni, I. (2009). Movement-specific repetition suppression in ventral and dorsal premotor cortex during action observation. Cerebral Cortex, 19(11), 2736-45. doi:10.1093/cercor/bhp049 
Marconi, B., Pecchioli, C., Koch, G., \& Caltagirone, C. (2007). Functional overlap between hand and forearm motor cortical representations during motor cognitive tasks. Clinical Neurophysiology, 118(8), 1767-75. doi:10.1016/j.clinph.2007.04.028

Mc Cabe, S. I., Villalta, J. I., Saunier, G., Grafton, S. T., \& Della-Maggiore, V. (2014). The relative influence of goal and kinematics on corticospinal excitability depends on the information provided to the observer. Cerebral Cortex, doi:10.1093/cercor/bhu029

Molenberghs, P., Cunnington, R., \& Mattingley, J. B. (2012). Brain regions with mirror properties: a meta-analysis of 125 human fMRI studies. Neuroscience and Biobehavioral Reviews, 36(1), 341-9. doi:10.1016/j.neubiorev.2011.07.004

Murata, A., Fadiga, L., Fogassi, L., Gallese, V., Raos, V., Rizzolatti, G. (1997). Object Representation in the Ventral Premotor Cortex ( Area F5 ) of the Monkey. Journal of Neurophysiology, 78(4), 2226-30.

Oldfield, R. (1971). The assessment and analysis of handedness: the Edin- burgh inventory. Neuropsychologia. 9,97-113.

Pascual-Leone, A., Manoach, D. S., Birnbaum, R., \& Goff, D. C. (2002). Motor cortical excitability in schizophrenia. Biological Psychiatry, 52(1), 24-31.

Raos, V., Umilta, M., Murata, A., Fogassi, L., \& Gallese, V. (2006). Functional Properties of GraspingRelated Neurons in the Ventral Premotor Area F5 of the Macaque Monkey. Journal of Neurophysiology, 95(2) 709-729. doi:10.1152/jn.00463.2005.

Rizzolatti, G., Fadiga, L., Gallese, V., \& Fogassi, L. (1996). Premotor cortex and the recognition of motor actions. Cognitive Brain Research, 3(2), 131-41.

Rizzolatti, G., \& Craighero, L. (2004). The mirror-neuron system. Annual Review of Neuroscience, 27, 169-92. doi:10.1146/annurev.neuro.27.070203.144230 
Rossini, P. M., Barker, A. T., Berardelli, A., Caramia, M. D., Caruso, G., Cracco, R. Q., et al. (1994). Noninvasive electrical and magnetic stimulation of the brain, spinal cord and roots: basic principles and procedures for routine clinical application. Report of an IFCN committee. Electroencephalogr Clin Neurophysiol. 91(2):79-92.

Saucedo Marquez, C.M., Ceux, T., Wenderoth, N. (2011). Attentional demands of movement observation as tested by a dual task approach. PLoS One, 6(11), e27292. doi: 10.1371/journal.pone.0027292

Senot, P., D’Ausilio, A., Franca, M., Caselli, L., Craighero, L., \& Fadiga, L. (2011). Effect of weightrelated labels on corticospinal excitability during observation of grasping: a TMS study. Experimental Brain Research, 211(1), 161-7. doi:10.1007/s00221-011-2635-x

Tucker, M., \& Ellis R. (1998). On the relations between seen objects and components of potential action. Journal of Experimental Psychology: Human Perception and Performance. 24(3):830-846.

Umilta, M. A., Brochier, T., Spinks, R. L., \& Lemon, R.N. (2007). Simultaneous recording of macaque premotor and primary motor cortex neuronal populations reveals different functional contributions to visuomotor grasp. Journal of Neurophysiology. 98(1):488-501.

Urgesi, C., Moro, V., Candidi, M., \& Aglioti, S. M. (2006). Mapping implied body actions in the human motor system. The Journal of Neuroscience, 26(30), 7942-9. doi:10.1523/JNEUROSCI.1289-06.2006

Urgesi, C., Candidi, M., Ionta, S., \& Aglioti, S. M. (2007). Representation of body identity and body actions in extrastriate body area and ventral premotor cortex. Nature Neuroscience, 10(1), 30-1. doi:10.1038/nn1815 
Figure 1. General setup.

A) Participants sat on a chair in front of a computer screen on which video-clips were displayed. To ensure complete relaxation, the right arm was comfortably positioned on an armrest during the entire experiment.

B) Schematic representation of the coil orientations and locations of transcranial magnetic stimulation. In experiment 1 and 2, single-pulse stimulations (130\% rMT) were applied over the primary motor cortex (M1). The stimulation location (coil position shown in green) represents the hotspot location of the first dorsal interosseus (FDI). In experiment 3 half of the stimulations over M1 (120\% rMT) were accompanied by a sub-threshold conditioning stimulus ( $80 \%$ rMT) applied $7 \mathrm{~ms}$ earlier over the ipsilateral premotor cortex (PMv, coil position shown in red). The stimulation site over PMv was localized based on mean Montreal Neurological Institute (MNI) coordinates -58, 13, 21 $(x, y, z)$ as indicated by a red dot in the lower panel. 
Figure 2. Schematic presentation of the video-clips showing the reaching, grasping and lifting of a round jar ( $9 \mathrm{~cm}$ diameter, $14.5 \mathrm{~cm}$ height) weighing $1.5 \mathrm{~kg}$ by an actor. In all 3 experiments, two types of video-clips were presented showing either a precision grip (PG) or a whole hand grip (WHG). During the whole hand grip, all fingers were used to grasp the lid of the jar, whereas for the precision grip, only the thumb and index finger grasped a small knob mounted on the top of the jar. In both clips, the right hand of an actor reached for the metal jar, grasped it with a whole hand or precision grip, and lifted it approximately $15 \mathrm{~cm}$ above the surface. TMS pulses are administered at crucial time points (frames surrounded by yellow boxes) while observing the unfolding of the grasping movement; more specifically during the reach $(2.0 \mathrm{~s})$, grasp (3.48 s) and lift phase (4.92 s).

A) In experiment 1 , single-pulse TMS is applied to measure M1 corticomotor excitability during the observation of 2 types of video clips (WHG and PG) at 3 phases of the unfolding action (reach, grasp, lift). Note that in this experiment, no precue providing information on the upcoming to-be-observed grasping action was provided to the observing subjects.

B) In experiment 2, single-pulse TMS is applied to measure M1 corticomotor excitability during the observation of 2 types of video clips (WHG and PG) at 2 phases of the unfolding action (reach and lift). In one half of the video-clips, a precue (white or blue screen) preceded the video-clip which provides information on the upcoming to-be-observed grasping action (PG, white precue; WHG, blue precue).

C) In experiment, paired-pulse TMS is applied to measure PMv-M1 interactions during the observation of 2 types of video clips (WHG and PG) at 3 phases of the unfolding action (reach, grasp, lift). Particularly, in half of the trials, stimulations over M1 were accompanied by a sub-treshold conditioning stimulus given 7 ms earlier over ipsilateral PMv. 
Figure 3. Results of Experiment 1 and 2. Evolution of MEPs during the observation of a whole hand grip (WHG) and precision grip (PG) shown as ratio scores (MEP ${ }_{\mathrm{WHG}} / \mathrm{MEP}_{\mathrm{PG}}$ ) separately for each movement phase (reach, grasp and lift) and muscle (FDI and ADM).

Values $>1$ indicate higher facilitation during the WHG, values $<1$ indicate larger facilitation during the PG. A ratio equal to 1 indicates no muscle-specific facilitation/inhibition.

A) Experiment 1: Muscle-specific facilitation is seen during the grasp and lift phase, particularly for the ADM. Since in experiment 1 , participants received no prior information regarding the to-beobserved grasping movement, no 'anticipatory' muscle-specific facilitation was observed during the reach phase, i.e., before movement initiation.

B) Experiment 2: Anticipatory' muscle-specific facilitation was observed during the reach phase, only for trials in which an informative precue regarding the upcoming to-be-observed grasping movement was provided (grey line). No such anticipatory muscle-specific facilitation was observed for trials in which no prior information was provided to the observing subjects (black line). During the lift phase, muscle-specific facilitation was observed for all trials (i.e., with (grey line) or without (black line) precue). Effects were most pronounced for the ADM muscle compared to the FDI.

\#: $p<0.05$ (a single sample t-test); +: $p<0.05$ (muscle main effect); *: $p<0.05$ (muscle by cue interaction). Vertical bars indicate SEM. 
Figure 4. Results of experiment 3.

A) The grey lines depict the evolution of MEPs obtained from trials in which single-pulse TMS is applied over M1, i.e. without a conditioning pulse over PMv. MEPs are shown as ratio scores (MEP ${ }_{\mathrm{WHG}} / \mathrm{MEP}_{\mathrm{PG}}$ ) separately for each phase (reach, grasp and lift) and muscle (FDI and ADM). Ratio scores $>1$ indicate higher facilitation during the WHG, values $<1$ indicate larger facilitation during the PG. A ratio equal to 1 indicates no muscle-specific facilitation/inhibition.

B) Similar to results from experiment 2 (Fig. 3B), anticipatory muscle-specific facilitation is seen during the reach phase. 'Online' muscle-specific facilitation during the unfolding of the action was only observed during the lift phase, not during the grasping phase.

C) Modulatory influences of PMv over M1 during movement observation. The bar plots depict for each muscle (FDI and ADM) and for each grasp type (WHG and PG) the changes in M1 facilitation from single-pulse TMS (SP) to paired pulse TMS (PP) (with conditioning stimulus over PMv). Ratio scores (MEP $\mathrm{sp} / \mathrm{MEP} \mathrm{PP}_{\mathrm{PP}}$ ), higher than 1 indicate facilitatory PMv-M1 interactions, negative ratio-scores indicate inhibitory PMv-M1 interaction. A ratio equal to 1 indicates no net influence of PMv over M1. Only during the observation of the grasp phase, not during reach or lift, a net facilitatory effect of PMv over M1 was measured in the ADM muscle. No other significant effects were observed for the ADM or FDI muscles.

\#: $p<0.05$ (single sample $t$-test); $++: p<0.01$ (muscle main effect); ${ }^{* *}: p<0.01$ (significant difference between the ratio MEPPP/MEP SP calculated for PG versus WHG). Vertical bars indicate SEM. 
A

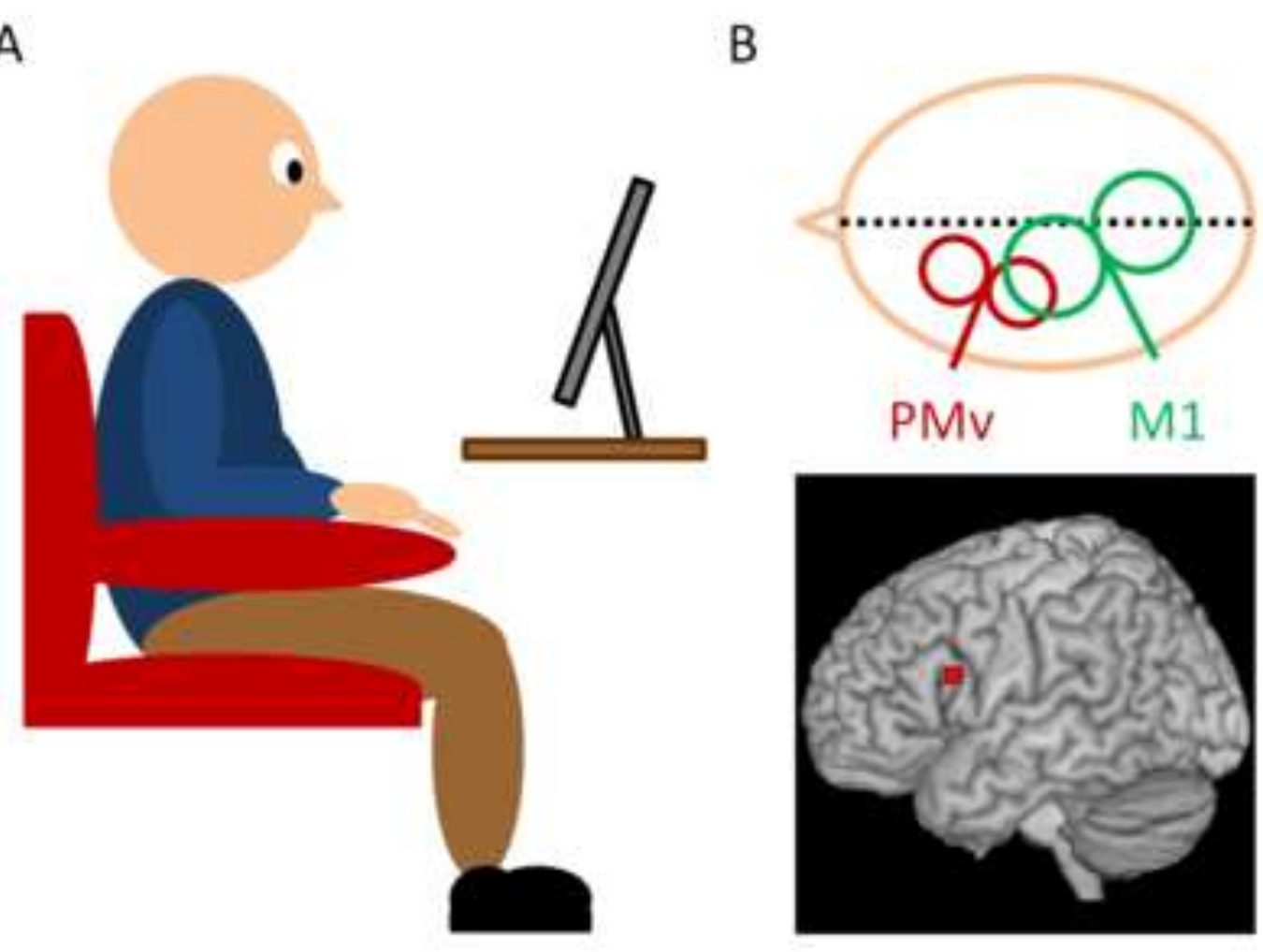

B
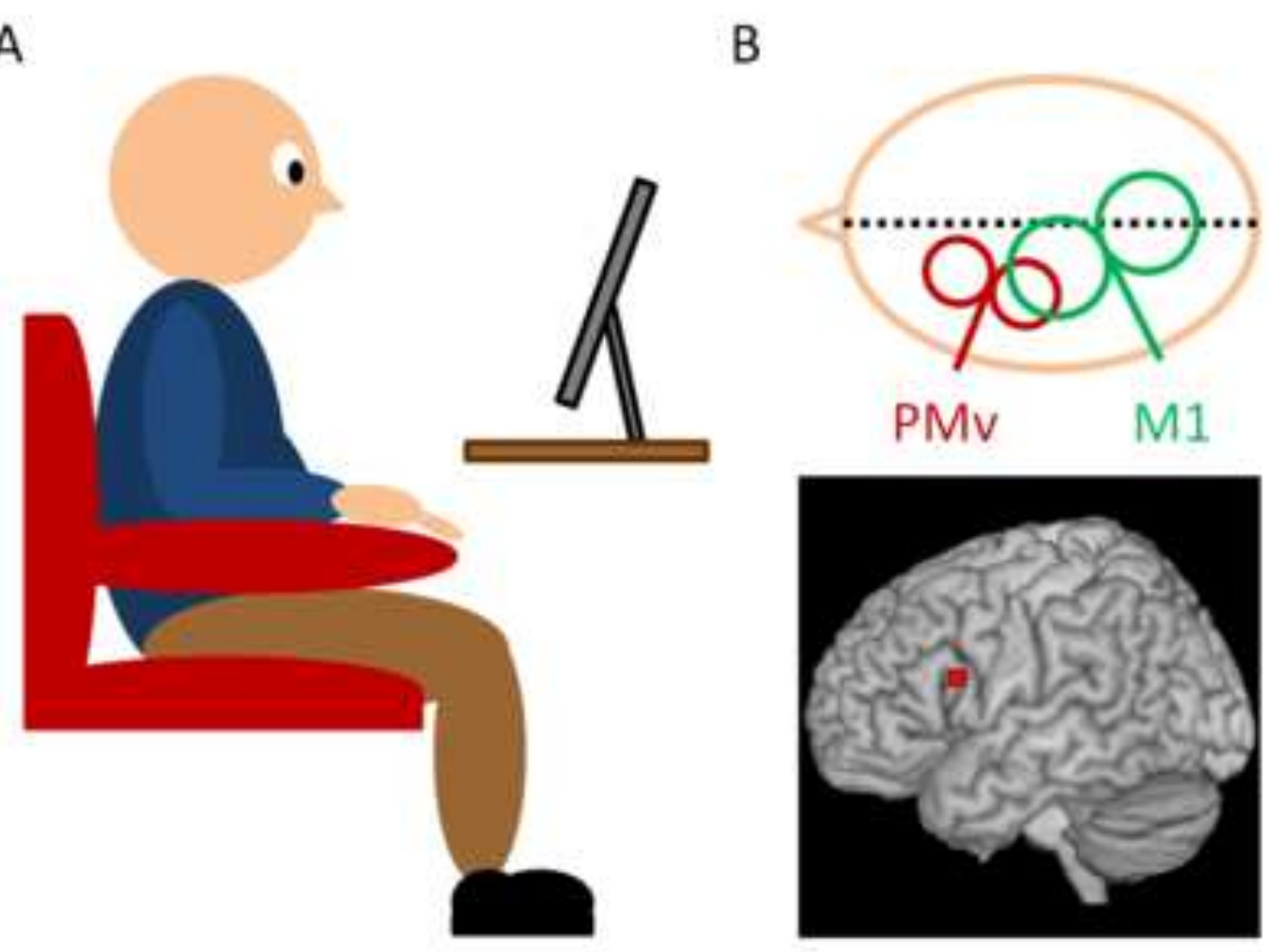

Revised Figure 1 
A

WHG
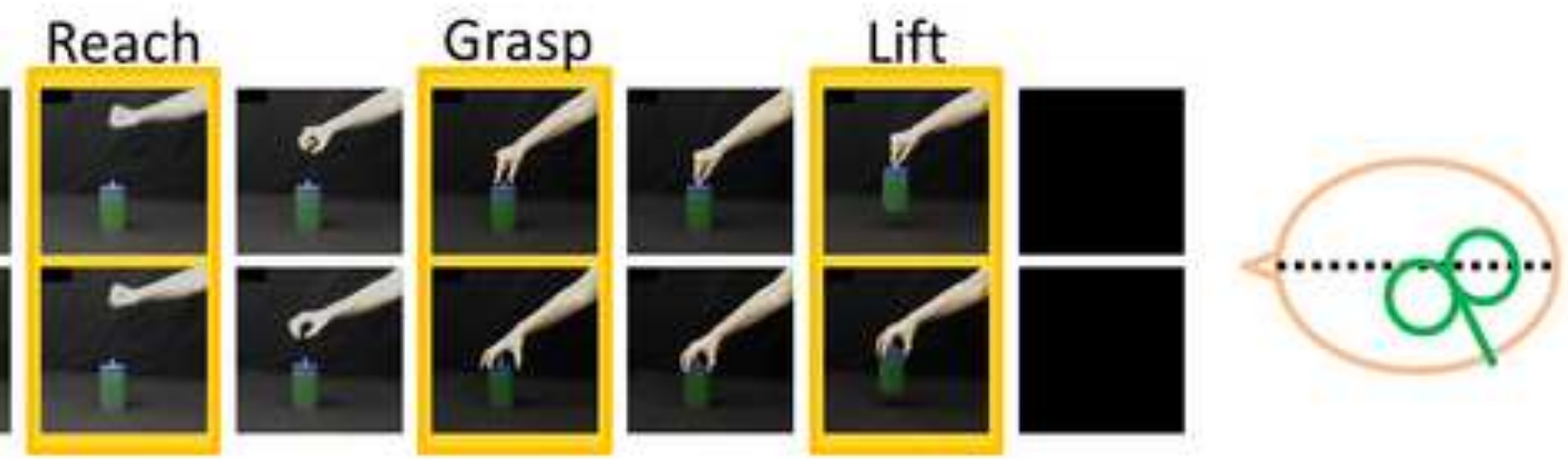

B
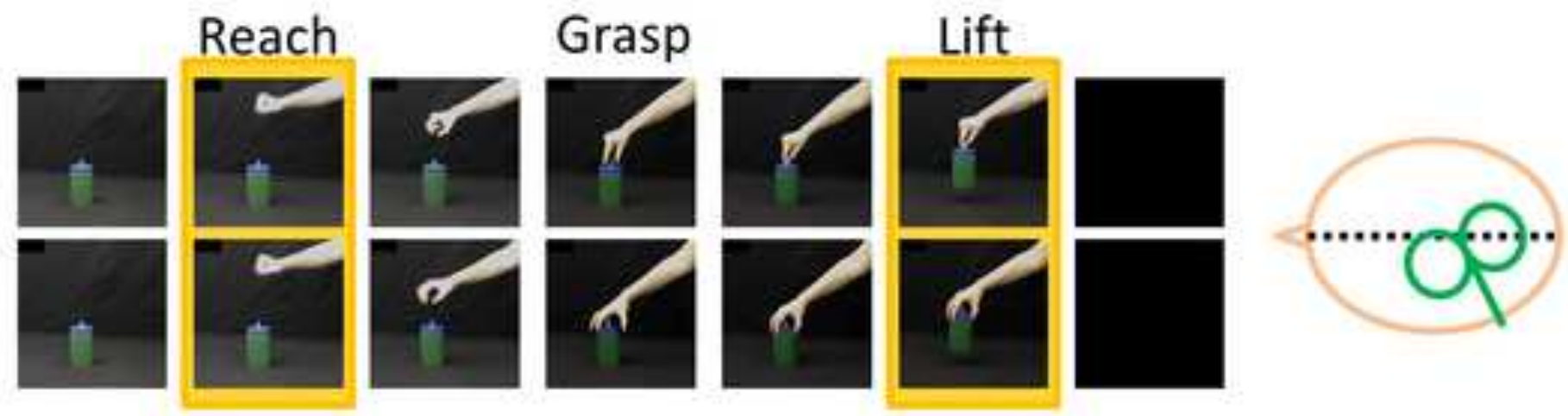

C
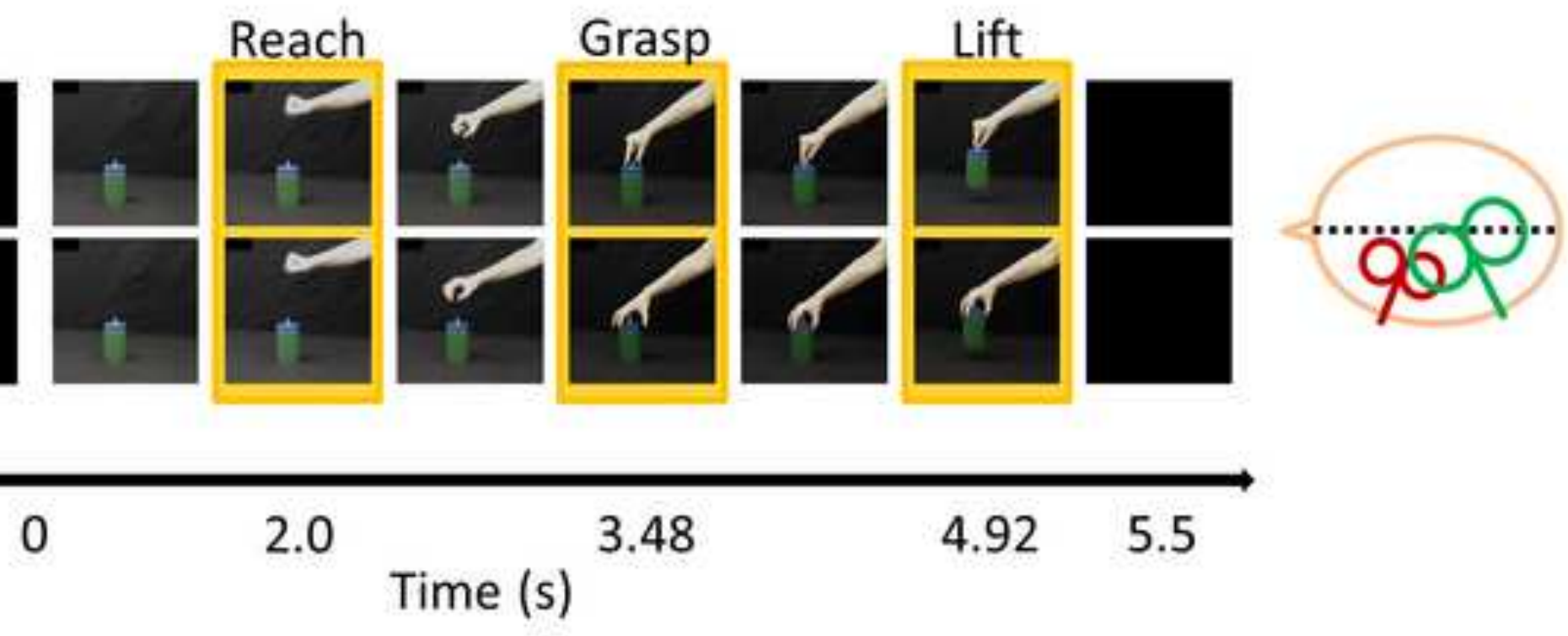

$4.92 \quad 5.5$ 
A

Reach

Grasp

Lift
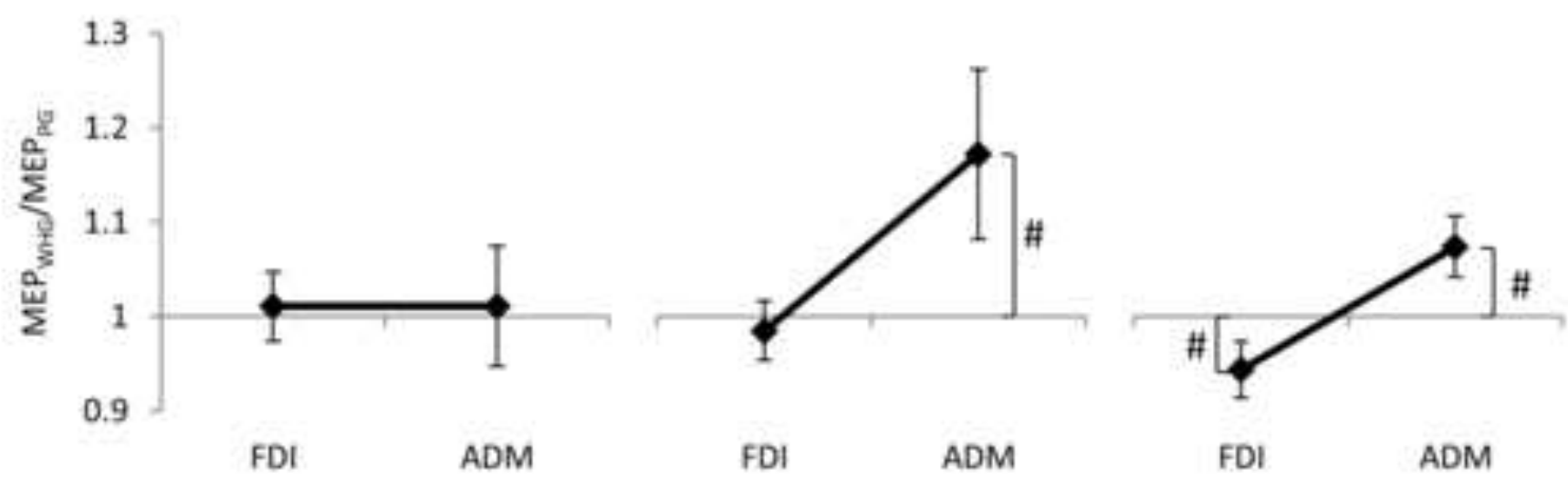

B
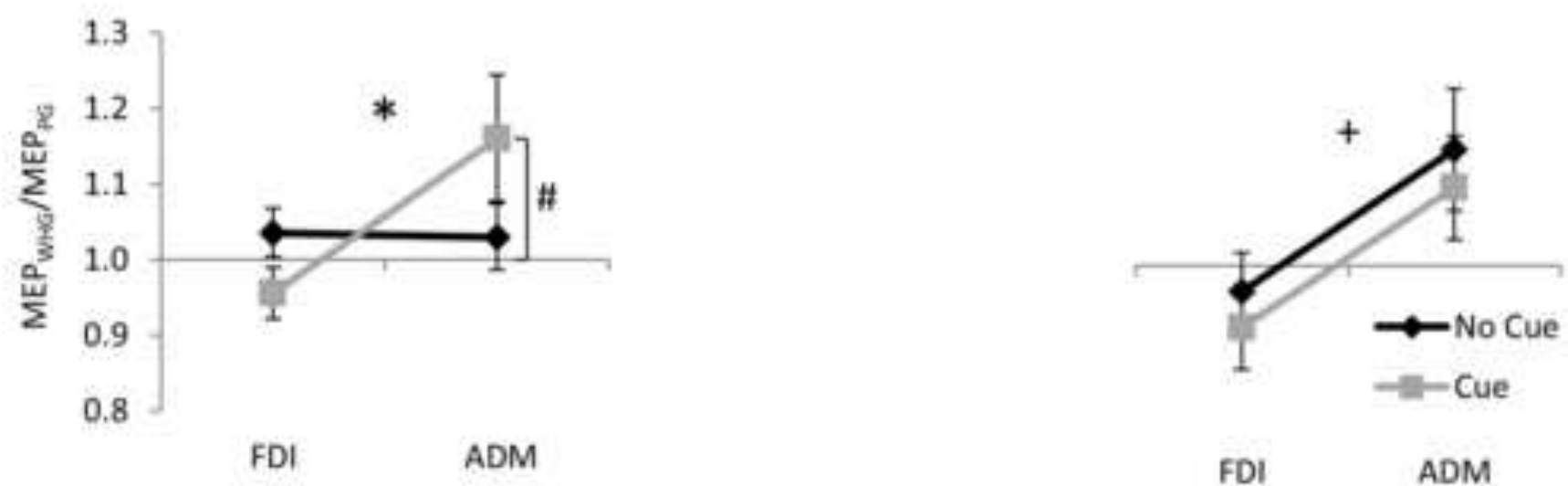

PG
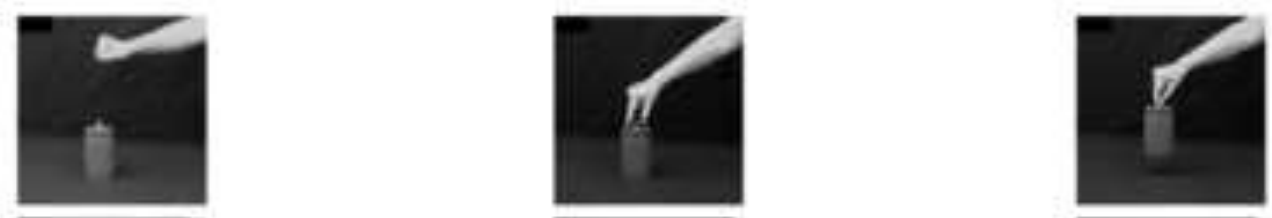

WHG
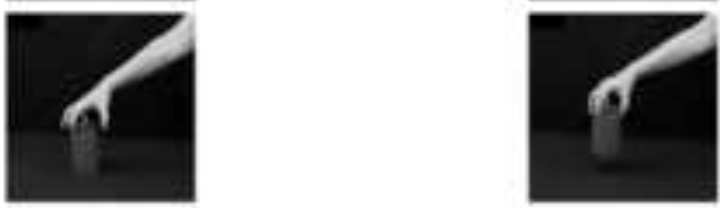
A

Reach

Grasp

Lift

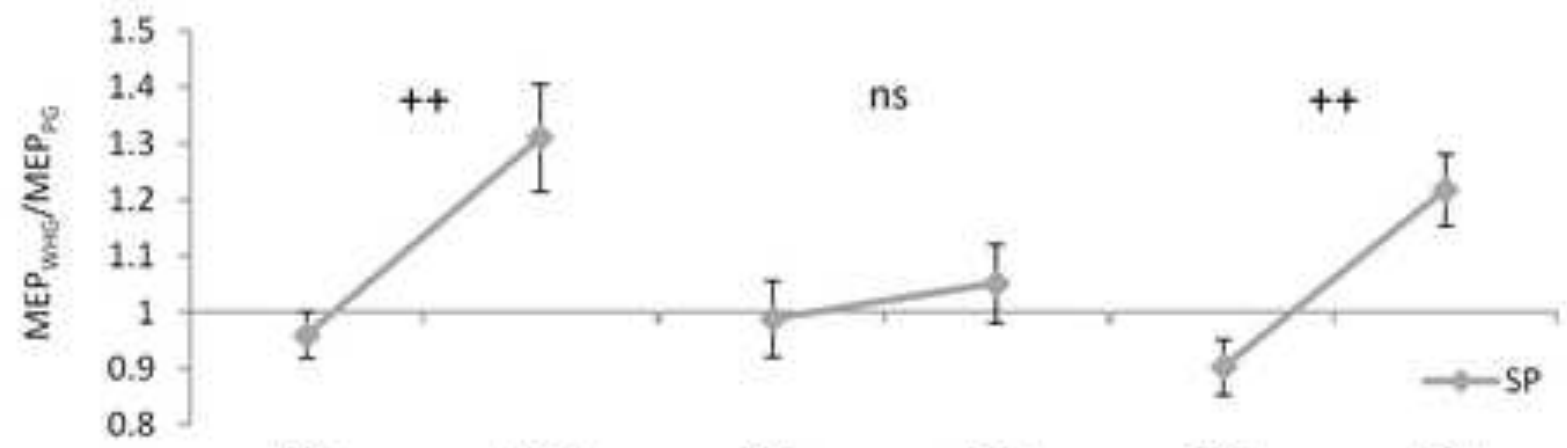

B
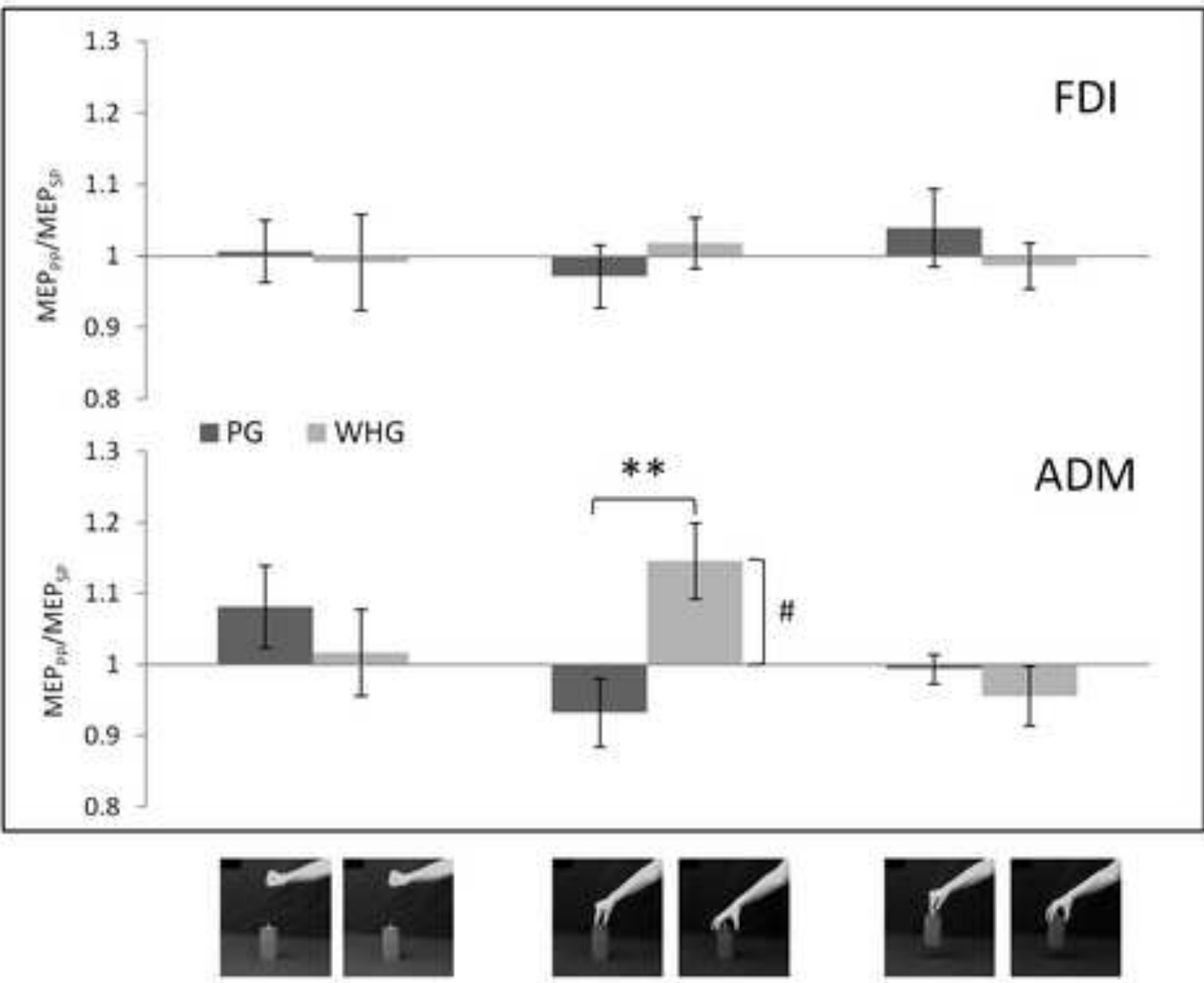